RM3-TH/00-21

\title{
Quark Masses on the Lattice: Light and Heavy
}

\author{
V. Lubicz ${ }^{\mathrm{a}}$ \\ a Dipartimento di Fisica, Università di Roma Tre and INFN, Sezione di Roma III, \\ Via della Vasca Navale 84, I-00146 Rome, Italy
}

I review the current status of lattice calculations of light and heavy quark masses. Significant progresses, in these studies, have been allowed by the introduction of improved actions and non-perturbative renormalization techniques. Current determinations of light quark masses are accurate at the level of $20 \%$, where the main source of uncertainty is represented by the quenching error. The determination of the bottom quark mass is accurate at the impressive level of $2 \%$. As final averages of lattice results, I quote $\bar{m}_{u d}(2 \mathrm{GeV})=(4.5 \pm 1.0) \mathrm{MeV}$, $\bar{m}_{s}(2 \mathrm{GeV})=(110 \pm 25) \mathrm{MeV}$ and $\bar{m}_{b}\left(\bar{m}_{b}\right)=(4.26 \pm 0.09) \mathrm{GeV}$.

\section{INTRODUCTION}

Calculations of quark masses are one of the most intensive subject of investigation for lattice QCD. An accurate determination of these parameters is in fact extremely important, for both phenomenological and theoretical applications. The charm and bottom masses, for instance, enter through the heavy quark expansion, the theoretical expressions of several cross sections and decay rates. From a theoretical point of view, an accurate determination of quark masses may give insights on the physics of flavour, revealing relations between masses and mixing angles, or specific textures in the quark mass matrix, which may originate from still uncovered flavour symmetries.

The values of quark masses cannot be directly measured in the experiments because quarks are confined inside the hadrons. On the other hand, quark masses are fundamental (free) parameters of the theory and, as such, they cannot be computed on the basis of purely theoretical considerations. The values of quark masses can be only determined by comparing the theoretical evaluation of a given physical quantity, which depends on quark masses, with the corresponding experimental value. Typically the pion, kaon and $\phi$ meson masses are used on the lattice to compute the values of the light quark masses, whereas the $b$ quark mass is determined by computing the mass of the $B$ or the $\Upsilon$ mesons. Different choices are all equivalent in principle, and the differences in the results, obtained by using different hadron masses as input parameters, give an estimate of the systematic error.

As all other parameters of the Standard Model lagrangian, quark masses can be defined as effective couplings, which are both renormalization scheme and scale dependent. A scheme commonly adopted for quark masses is the $\overline{\mathrm{MS}}$ scheme, with a renormalization scale chosen in the short-distance region in order to make this quantity accessible to perturbative calculations. It is a common practice to quote the values of the light quark masses at the renormalization scale $\mu=2 \mathrm{GeV}$, whereas the heavy quark masses are usually given at the scale of the quark mass itself, e.g. $\bar{m}_{b}\left(\bar{m}_{b}\right)$. This convention will be followed in the present review.

At Lattice 99, no plenary talk was dedicated to review lattice calculations of quark masses. For this reason, I will mainly report on results presented in the last two years. With respect to previous determinations, significant progresses in controlling the systematic errors have been allowed by the extensive implementation of non-perturbative $\mathcal{O}(a)$-improvement [1] and non-perturbative renormalization techniques [2, 3. The effect of these tools, on lattice calculations of quark masses, will be discussed in sec. 2 . 
The recent determinations of light quark masses will be presented in sec. 3, and of the $b$-quark mass in sec. 4. Some comments on the determination of the charm quark mass will be done in sec. 5 , where I will also summarize the lattice "world averages" of quark masses and present my conclusions.

\section{DEFINITIONS AND THEORETICAL PROGRESSES}

\subsection{Quark Masses and $\mathcal{O}(a)$-improvement}

Non perturbative definitions of quark masses are provided by the chiral Ward identities (WI) of QCD [4]. These definitions allow us to express the renormalized quark mass, in a given scheme and at a given renormalization scale, in terms of lattice renormalization constants and bare quantities (action parameters, matrix elements or correlation functions). Three independent definitions have been proposed so far which are all equivalent in principle, in the sense that they lead to the same value of the renormalized mass. Let us discuss these definitions in some details.

1) Vector Ward Identity definition:

In terms of renormalized quantities, the chiral vector WI reads:

$\nabla_{\mu}\left\langle\alpha\left|\hat{V}_{\mu}\right| \beta\right\rangle=\left(m_{1}(\mu)-m_{2}(\mu)\right)\langle\alpha|\hat{S}(\mu)| \beta\rangle$

where $|\alpha\rangle$ and $|\beta\rangle$ are arbitrary external states. Vector current conservation, within the lattice regularization, implies a lattice version of the vector WI, from which the following definition of the renormalized quark mass, in terms of the (bare) Wilson parameter, can be derived:

$m(\mu)=Z_{S}^{-1}(a \mu) m=Z_{S}^{-1}(a \mu) \frac{1}{2 a}\left(\frac{1}{K}-\frac{1}{K_{c}}\right)(2)$

An $\mathcal{O}(a)$-improved definition is obtained with the simple replacement [1]:

$m \rightarrow m\left(1+a b_{m} m\right) \quad, \quad\left(b_{m}=b_{S} / 2\right)$

2) Axial Ward Identity definition:

In the Wilson formulation of lattice QCD, axial symmetry is explicitly broken. The axial WI can be then imposed only for renormalized quantities, and, in the case of degenerate masses, takes the form:

$\nabla_{\mu}\left\langle\alpha\left|\hat{A}_{\mu}\right| \beta\right\rangle=2 m(\mu)\langle\alpha|\hat{P}(\mu)| \beta\rangle$

From this identity, an alternative definition of the quark mass is derived:

$m(\mu)=\frac{Z_{A}}{Z_{P}(a \mu)} m^{\mathrm{AWI}}=\frac{Z_{A}}{Z_{P}(a \mu)} \frac{\nabla_{\mu}\left\langle\alpha\left|A_{\mu}\right| \beta\right\rangle}{2\langle\alpha|P| \beta\rangle}(5)$

In this case, $\mathcal{O}(a)$-improvement is achieved with the replacement [1]:

$$
\begin{aligned}
m^{\mathrm{AWI} \rightarrow} & {\left[1+a\left(b_{A}-b_{P}\right) m\right] \cdot } \\
& \frac{\nabla_{\mu}\left\langle\alpha\left|\left(A_{\mu}+a c_{A} \nabla_{\mu} P\right)\right| \beta\right\rangle}{2\langle\alpha|P(\mu)| \beta\rangle}
\end{aligned}
$$

In the absence of explicit chiral symmetry breaking induced by the Wilson term in the action, the vector and axial vector WI definitions are not independent anymore. This is the case, for instance, of staggered fermions and of the proposed realizations of Ginsparg-Wilson fermions (domain wall and overlap fermions).

3) Definition from the Quark Propagator: This relatively new method has been proposed by APE [5]. It is based on the study of the quark propagator, computed in a fixed gauge, at large momentum. In this limit, an OPE can be derived for the trace of the quark propagator which, up to known logarithmic corrections, reads [6] :

$\operatorname{Tr} \hat{S}(p) \simeq \frac{m}{p^{2}}+\frac{4 \pi \alpha_{s}}{3 p^{4}}\langle\bar{\psi} \psi\rangle+\mathcal{O}\left(1 / p^{6}\right)$

The leading term of this expansion is proportional to the renormalized quark mass. The Wilson coefficient of this term is fixed by an axial WI which, in RI-MOM scheme, implies [5]:

$m(\mu)=\frac{1}{12} \operatorname{Tr}\left[\hat{S}^{-1}(p, \mu)\right]_{p^{2}=\mu^{2}}$

Note that the relevant renormalization constant, in this case, is the quark field renormalization constant $Z_{q}$. Cancellation of leading $\mathcal{O}(a)$ effects is obtained by improving the quark field. Since this field is a non gauge-invariant operator, and the quark propagator is an off-shell correlation function, improvement involves mixing with 
both non gauge-invariant operators and operators vanishing by the equation of motion. The $\mathcal{O}(a)$-improvement of the quark field is obtained through the replacement [7]:

$$
q \rightarrow\left(1+a b_{q} m\right)\left[1+a c_{q}^{\prime}\left(\not D+m_{0}\right)+a c_{\mathrm{NGI}} \not \partial\right] q
$$
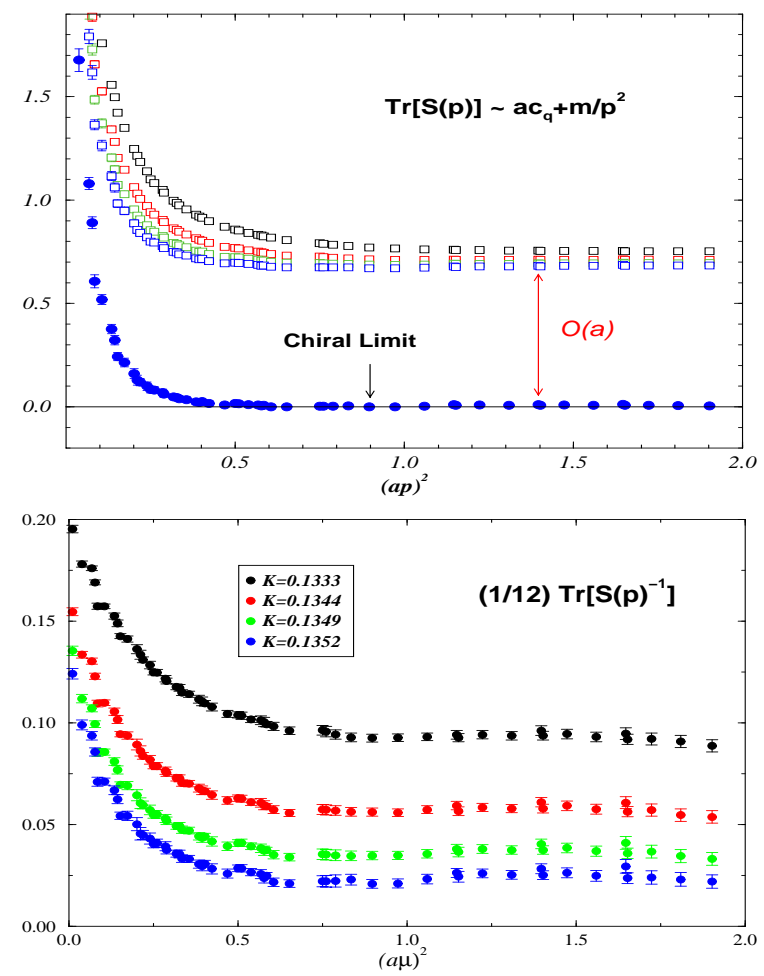

Figure 1. Large momentum behaviour of the lattice quark propagator. The upper figure shows the large $\mathcal{O}(a)$ term which has to be subtracted before extracting the subleading contribution proportional to the quark mass. The behaviour of the inverse quark propagator, after the subtraction, is shown below. The results have been obtained by APE [5] using the non-perturbatively $\mathcal{O}(a)$-improved Wilson action at $\beta=6.2$.

In the determination of quark masses with the propagator method, the improvement procedure is a crucial requirement. The reason is that the OPE of the quark propagator on the lattice is dominated by a large lattice artifact. From eq. (9) one finds, in the large momentum limit and up to small logarithmic corrections [5]:

$\frac{1}{12} \operatorname{Tr} S(p) \simeq-2 a c_{q}^{\prime}+2 a c_{\mathrm{NGI}} Z_{q} \equiv a c_{q}$

The lattice quark propagator does not vanish at large $p^{2}$, as dictated in the continuum limit by eq. (7), but it is dominated by terms of $\mathcal{O}(a)$. This is illustrated in fig. 1. The numerical study of the propagator at large $p^{2}$, in the chiral limit, provides the value of the coefficient $c_{q}$, which can thus be subtracted before computing the subleading contribution to the OPE proportional to the quark mass.

The improved calculations of quark masses, with the vector and axial-vector WI methods, require the knowledge of several coefficients, $c_{A}$, $b_{A}, b_{S}$ and $b_{P}$, besides the coefficient $c_{S W}$ of the clover term in the action. Non-perturbative determinations of these coefficients have been performed in refs. [1, 8, 9]. One finds that the effect of improvement, in the determination of quark masses, is quite significant, even for light quarks. In the case of the strange quark mass, for instance, an estimate of the $\mathcal{O}(a)$ effects, with unimproved Wilson fermions, has been performed by Bhattacharya and Gupta [10] at Lattice 97 , based on the analysis of the continuum extrapolation. They find that discretization errors, at two typical values of the lattice coupling, $\beta=6.0$ and $\beta=6.2$, are of the order of $25 \%$ and $18 \%$ respectively. These estimates can be compared with those obtained with nonperturbatively $\mathcal{O}(a)$-improved Wilson fermions, from a recent study by ALPHA-UKQCD [11. In this case, residual discretization effects are reduced at the level of $7 \%$ and $3 \%$ respectively. Implementation of $\mathcal{O}(a)$-improvement is therefore a crucial ingredient to significantly increase the accuracy of lattice calculations of quark masses.

\subsection{Non-perturbative Renormalization}

Another potential source of systematic errors, in lattice calculations of quark masses, is introduced by the truncation of the perturbative expansion in the evaluation of the quark mass renormalization constant. Depending on the definition considered for the bare mass, this constant is given by $Z_{S}^{-1}$, with the vector WI method, 
Table 1

Values of the strange quark mass, $\bar{m}_{s}(2 \mathrm{GeV})$, in $\mathrm{MeV}$, as obtained by computing the quark mass renormalization constant either in one loop (boosted) perturbation theory (PT) or using the RI-MOM non-perturbative method (NP). The relative difference between the two determinations is also shown.

\begin{tabular}{lccccc}
\hline Action & $\beta$ & $\bar{m}_{s}[\mathrm{PT}]$ & $\bar{m}_{s}[\mathrm{NP}]$ & $\Delta$ & \\
\hline \multirow{2}{*}{ NP-CLOVER } & 6.2 & $95(10)^{\dagger}$ & $109(11)$ & $15 \%$ & APE 98 [13] \\
& 6.0 & $81(9)^{\ddagger}$ & $111(13)$ & $37 \%$ & \\
KOGUT-SUSSKIND & 6.2 & $81(4)$ & $114(5)$ & $43 \%$ & \\
& 6.4 & $83(4)$ & $110(8)$ & $36 \%$ & JLQCD 99 [14] \\
DOMAIN WALL & 6.0 & $106(8)(14)^{*}$ & $130(11)(18)$ & $23 \%$ & RBC 99 [15] \\
\hline
\end{tabular}

$\dagger$ From vector WI; ${ }^{\ddagger}$ from axial WI. ${ }^{*}$ The error is my estimate from the RBC data.

$Z_{A} / Z_{P}$, with the axial WI method, or the quark field renormalization constant $Z_{q}$ with the propagator method. In perturbation theory, all these quantities are known only at one loop. In the last two years, however, in most of lattice quark masses calculations, the systematic error associated with the evaluation of the renormalization constant has been reduced to a negligible amount by the use of non-perturbative renormalization techniques [2,3].

Non-perturbative renormalization is reviewed by Sint at this conference 12. For this reason, I will only discuss here those particular aspects of this topic which are more closely related to the determination of quark masses.

To show the impact of non-perturbative renormalization in lattice calculations of quark masses, I present in table 1 the values of the strange quark mass, as obtained by computing the quark mass renormalization constant either in one-loop (boosted) perturbation theory or with the RIMOM non-perturbative method of ref. [2]. For illustrative purposes, the results of three calculations are considered, which employed three different fermionic actions: non-perturbative Clover, Kogut-Susskind and domain wall fermions. As can be seen from table 1, the difference between the results obtained with perturbative and nonperturbative renormalization is rather large. Depending on the action, the value of the coupling constant and the specific renormalization constant $\left(Z_{S}\right.$ or $Z_{P}$ for Wilson fermions), the relative error varies in the range between $\sim 15 \%$ and $\sim 40 \%$. Moreover, at least in the case of staggered fermions, this error decreases as one get closer to the continuum limit, but at a very slow rate. Therefore, non-perturbative renormalization is required to improve the accuracy of lattice calculations of quark masses at a significant level (below 15\%).

I believe that a more detailed discussion deserves the non-perturbative calculation of the pseudoscalar renormalization constant, $Z_{P}$, in the RI-MOM scheme. Although rather technical, this discussion is of relevance for the determination of quark masses with the axial WI method. Within the non-perturbative approach of ref. [2], $Z_{P}$ is evaluated from the amputated correlation function of the pseudoscalar density, computed between external off-shell quark states of momentum $p$. This function, $\Gamma_{P}(p)$, once conveniently projected onto the matrix $\gamma_{5}$, is related to the trace of the inverse quark propagator by the axial WI: $m \widehat{\Gamma}_{P}(p)=\operatorname{Tr} \hat{S}^{-1}(p)$. The OPE of the quark propagator, given in eq. (7), thus implies:

$\widehat{\Gamma}_{P}(p) \simeq C_{1}\left(p^{2}\right)+C_{2}\left(p^{2}\right) \frac{\langle\bar{\psi} \psi\rangle}{m p^{2}}+\mathcal{O}\left(1 / p^{4}\right)$

The renormalization constant $Z_{P}$ is determined from the coefficient function $C_{1}$. Eq. (11) shows that the first power correction, which vanishes at large momentum, is however divergent in the chiral limit. Physically, this divergence is introduced by the coupling of the pseudoscalar density to the pion field, which is the Goldstone boson of the spontaneously broken chiral symmetry. It has 
been emphasized, particularly in ref. [16], that, because of the singular behaviour of this term in the chiral limit, its contribution to the correlation function $\Gamma_{P}(p)$ may not be suppressed enough at large $p^{2}$, at least for those values of $p^{2}$ in actual calculations at which discretization effects are still under control. This may affect the determination of $Z_{P}$ and, according to ref. [16], this systematic error may in fact be the reason of the large discrepancy between the perturbative and the non-perturbative determination of $Z_{P}$. Since $Z_{m} \sim Z_{P}^{-1}$, the observation of ref. [16] is of relevance for lattice calculations of quark masses.

I believe that the warning of ref. [16] deserves some consideration and a careful investigation. I would like to mention, therefore, three different methods which can be used to verify whether the contribution of the Goldstone pole, in the actual calculation of $Z_{P}$, is suppressed at the required level. To study this problem, I will use the same APE data which have been used for the non-perturbative calculation of the renormalization constant of bilinear quark operators $\left(Z_{V}, Z_{A}\right.$, $Z_{S}, Z_{P}$ and $Z_{T}$ ) with the non-perturbative Clover action, at $\beta=6.2$ [17]. The first method is based on the study of the scale dependence of the ratio $Z_{P} / Z_{S}$, computed non-perturbatively in the RIMOM scheme. In the large momentum region, where power corrections are expected to be negligible, this ratio should exhibit a plateau, since the two renormalization constants have equal anomalous dimensions. This ratio is shown in fig. 2 , and in the large- $p^{2}$ region a plateau is clearly visible. This suggests that all power corrections, including the contribution of the Goldstone pole, are suppressed enough. The second method is based on the study of the renormalization group invariant combination $Z_{P}(a \mu) / C(a \mu)$, where the running factor $C(a \mu)$, computed in perturbation theory at the NLO, expresses the predicted logarithmic scale dependence of the renormalization constant. Again, in the region where nonperturbative power corrections are suppressed enough, the ratio $Z_{P}(a \mu) / C(a \mu)$ should behave as a constant. Indeed, this constant behaviour is observed in fig. 2. Finally, the most efficient way to get rid of the Goldstone boson contribution in the calculation of $Z_{P}$ (but the method can be ea-

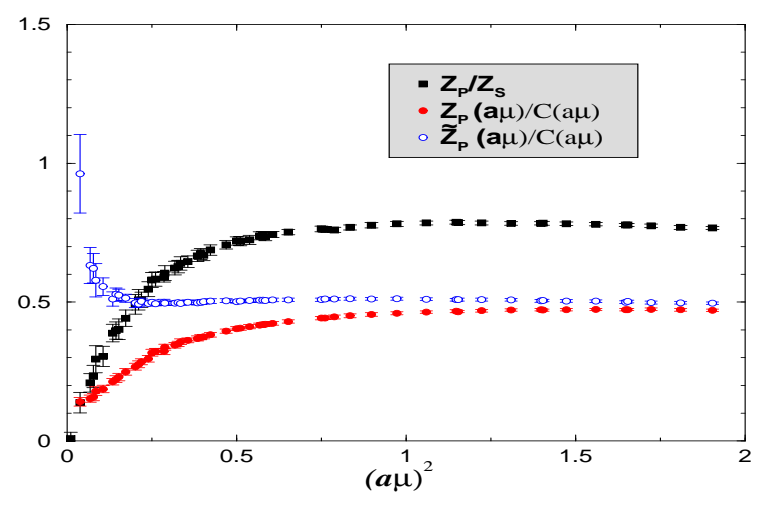

Figure 2. The ratios $Z_{P} / Z_{S}, Z_{P}(a \mu) / C(a \mu)$ and $\widetilde{Z}_{P}(a \mu) / C(a \mu)$ (see text for details) as a function of the scale $(a \mu)^{2}$.

sily adapted to the calculation of other renormalization constants) has been recently suggested in ref. [18]. The idea is to consider a new definition of $Z_{P}$, which I will denote with $\widetilde{Z}_{P}$, involving two flavours of non-degenerate quarks:

$\frac{\widetilde{Z}_{P}(\mu)}{Z_{q}(\mu)}=\frac{m_{1}-m_{2}}{m_{1} \Gamma_{P}\left(\mu, m_{1}\right)-m_{2} \Gamma_{P}\left(\mu, m_{2}\right)}$

On the r.h.s. of eq. (12), the limit $m_{1}, m_{2} \rightarrow 0$ is understood. For $m_{2}=0$ (and at large values of the scale), the above definition reduces to the standard RI-MOM definition of $Z_{P}$. However, one immediately realizes that, at the leading order in the quark mass, the Goldstone boson contribution of eq. (11) cancels in the definition of $\widetilde{Z}_{P}$, which is thus well behaved in the chiral limit. To the calculation of $\widetilde{Z}_{P}$, the warning of ref. 16 does not apply anymore. The ratio $\widetilde{Z}_{P}(\mu) / C(a \mu)$ is plotted in fig. 2 as a function of the scale. One can see, in this case, that the desired constant behaviour is reached much faster than with the ratio $Z_{P}(\mu) / C(a \mu)$. Moreover, the two ratios converge to the same value at large scales, within less than $5 \%$, an uncertainty which is of the same order of the other systematic errors involved in the calculation. This analysis supports the assumption of an adequate suppression of the Goldstone pole contribution in previous calculations of $Z_{P}$.

A significant accuracy in the non-perturbative determination of the quark mass renormalization constant has been achieved by ALPHA [3], within 
the Schrödinger functional approach. Besides determining the value of mass renormalization constant at a low hadronic scale, ALPHA has also computed, non-perturbatively, the running factor $C(\mu)$ over a large region extending from the low hadronic scale up to a very high scale. This calculation, which is based on a recursive finite-size scaling technique, allows a fully non-perturbative determination of the renormalization group invariant quark mass. The non-perturbative calculation of $C(\mu)$ turns out to be in very good agreement with the predictions of NLO perturbation theory. The advantage of this approach is that the matching factor, relating the renormalization group invariant mass to the $\overline{\mathrm{MS}}$ mass, is known at $\mathrm{N}^{4} \mathrm{LO}$ in perturbation theory 19,20 . The remaining perturbative uncertainty in this determination is thus reduced at a completely negligible level. The ALPHA calculation of the mass renormalization constant has been discussed by Sint at this conference, and I refer to his review for further details [12].

\section{LIGHT QUARK MASSES}

\subsection{Quenched Calculations}

The determination of light quark masses is, at present, one of the main fields of activity of lattice QCD simulations. This is illustrated in fig. 3, which presents a compilation of all lattice results for the strange quark mass, in the quenched approximation, starting from the first lattice prediction for $\bar{m}_{s}$ obtained with a NLO accuracy [21. These results have been produced by using different actions, different orders of improvement, different renormalization techniques and different choices of the input parameters, used to fix both the scale and the strange quark mass itself (either $m_{K}$ or $m_{\phi}$ ). In spite of these differences, we find good consistency among the several determinations. In addition, all the results, with the possible exception of the rather large value obtained by SESAM [22], suggest an interval of allowed values for the strange quark mass which is smaller than the one quoted by the particle data group (PDG) [23], indicated by the band in the figure. One can also observe that the lattice determinations of the strange quark mass cluster close to

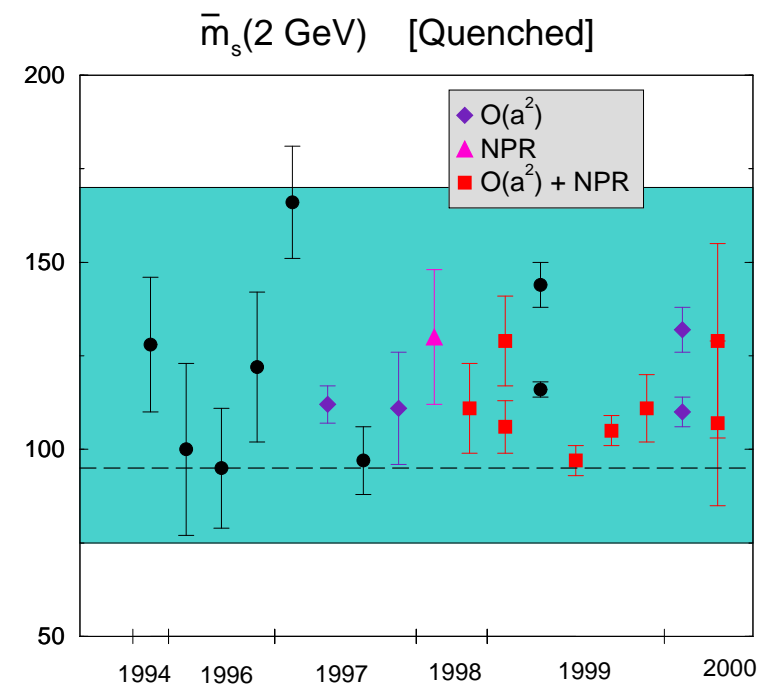

Figure 3. Results of all lattice calculations of the strange quark mass obtained, in the quenched approximation, since 1994. The band shows the estimate of $\bar{m}_{s}$ quoted by the particle data group. The dashed line indicates the unitarity lower bound on $\bar{m}_{s}$.

the unitarity lower bound, $\bar{m}_{s} \gtrsim 100 \mathrm{MeV}$ [24], obtained by using unitarity and the positivity of the pseudoscalar spectral function. Finally, the results which are plotted on the same vertical line in the figure have been obtained by using either the kaon or the $\phi$ meson mass as input parameter. It has been shown that a systematic difference of approximately $20 \%$ is found between the two determinations within the quenched approximation.

In order to derive an average value for the strange quark mass, I will concentrate in the following on the more recent and accurate results, which are given in table 2 and shown in fig. 1 with full circles. In this case, only the results obtained by using $m_{K}$ as experimental input have been considered.

The comparison among the results presented in table 2 becomes more significant if one tries to correct them by taking into account for the different systematic errors involved in the calcu-

\footnotetext{
${ }^{1}$ The $\mathrm{N}^{2} \mathrm{LO}$ bound of ref. 24] slightly decreases to $\bar{m}_{s} \gtrsim$ $95 \mathrm{MeV}$, when the $\mathrm{N}^{3} \mathrm{LO}$ perturbative corrections to the pseudoscalar spectral function are taken into account 25].
} 
Table 2

Quenched lattice results for the strange quark mass obtained by using in input the value of the kaon mass. Details concerning the action, the value of the lattice spacing, the renormalization procedure and the perturbative accuracy are also given.

\begin{tabular}{|c|c|c|c|c|c|c|}
\hline & Action & $a^{-}$ & & $\overline{Z_{m}}$ & $\overline{\mathrm{PT}}$ & $\bar{m}_{s}(2 \mathrm{GeV}) / \mathrm{MeV}$ \\
\hline APE 98 |13| & NP-Clover & $\sim 2.7 \mathrm{GeV}$ & $\left(m_{\rho}\right)$ & NP-RI & $\mathrm{N}^{2} \mathrm{LO}$ & $111 \pm 12$ \\
\hline JLQCD 9914 & KS & $a \rightarrow 0$ & $\left(m_{\rho}\right)$ & NP-RI & $\mathrm{N}^{2} \mathrm{LO}$ & $106 \pm 7$ \\
\hline CP-PACS 99 [26] & Wilson & $a \rightarrow 0$ & $\left(m_{\rho}\right)$ & $\mathrm{PT}$ & NLO & $116 \pm 3$ \\
\hline ALPHA-UKQCD 99 & NP-Clover & $a \rightarrow 0$ & $\left(r_{0}, f_{K}\right)$ & NP-SF & $\mathrm{N}^{4} \mathrm{LO}$ & $97 \pm 4$ \\
\hline QCDSF 99 & NP-Clover & $a \rightarrow 0$ & $\left(r_{0}\right)$ & NP-SF & $\mathrm{N}^{4} \mathrm{LO}$ & $105 \pm 4$ \\
\hline APE 99 [5] & NP-Clover & $\sim 2.7 \mathrm{GeV}$ & $\left(m_{\rho}\right)$ & NP-RI & $\mathrm{N}^{3} \mathrm{LO}$ & $111 \pm 9$ \\
\hline CP-PACS 00 [28] & MF-Clover & $a \rightarrow 0$ & $\left(m_{\rho}\right)$ & PT & NLO & $110_{-4}^{+3}$ \\
\hline RBC 00 29 & DWF & $\sim 1.9 \mathrm{GeV}$ & $\left(m_{\rho}\right)$ & NP-RI & $\mathrm{N}^{3} \mathrm{LO}$ & $\begin{array}{l}110 \pm 2 \pm 22^{\dagger} \\
105 \pm 6 \pm 21 \ddagger\end{array}$ \\
\hline
\end{tabular}

${ }^{\dagger}$ From vector WI; ${ }^{\ddagger}$ from axial WI.

QUENCHED

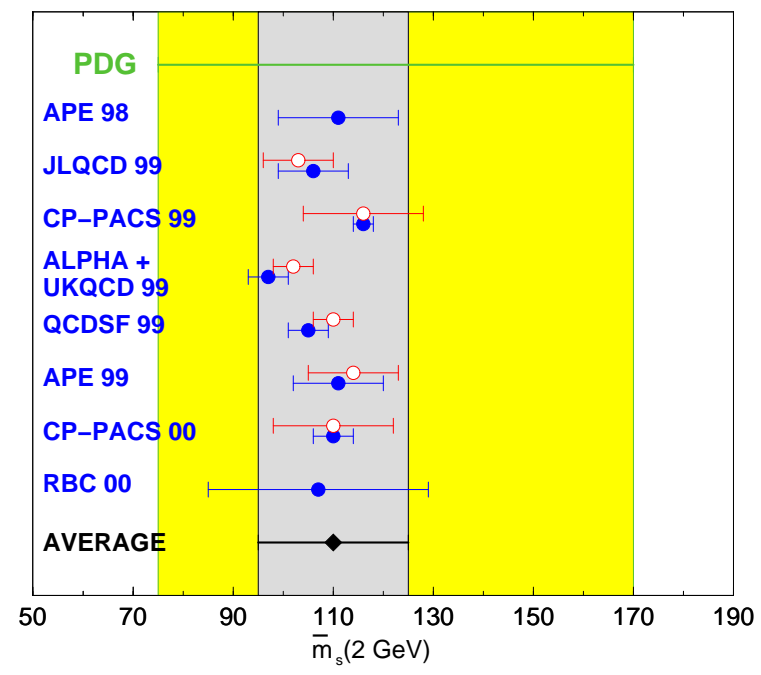

Figure 4. Values of the strange quark mass obtained from recent lattice calculations in the quenched approximation (full circles). Empty circles denote the values and errors obtained by the author attempting to correct for the different systematics.

lations. In this way, I obtain the estimates shown in fig. 1⿴囗十 as empty circles.

All results in table 2 have been obtained adopting a non-perturbative renormalization technique, with the only exceptions of the two CPPACS determinations 26, 28, in which the quark mass renormalization constant has been evalu- ated using one-loop perturbation theory. An additional uncertainty, due to the use of perturbation theory, should then be added to these results, to account for the corresponding systematic error. This uncertainty, however, is difficult to be quantified, because two different renormalization constants $\left(Z_{S}^{-1}\right.$ and $\left.Z_{A} / Z_{P}\right)$ have been used in the calculation and, moreover, non-perturbative determinations of renormalization constants with the Iwasaki action used in ref. 228] have not been performed yet. In fig. A, a systematic error of $10 \%$ has been added to the CP-PACS results. This error, however, may be underestimated, according to the analysis of the previous section (see table 1).

The values of the strange quark mass by ALPHA-UKQCD [11] and QCDSF 27] have been obtained by fixing the lattice spacing from the value of the phenomenological parameter $r_{0}$ [32], rather than from $m_{\rho}$ as in all other determinations. QCDSF finds that the choice of $r_{0}$ is roughly equivalent to using $m_{\rho}$, while ALPHAUKQCD finds that this is equivalent to setting the scale from $f_{K}$. ALPHA-UKQCD also estimates that a $10 \%$ higher value of $\bar{m}_{s}$ would have been obtained by fixing the scale from the value of the nucleon mass. Since the scale determined from $m_{\rho}$ turns out to be typically in between the values obtained from $f_{K}$ and $m_{N}$, in order to compare with the other determinations I have increased by $5 \%$ in fig. 1 the values of $\bar{m}_{s}$ obtained 
by ALPHA-UKQCD and QCDSF.

The other corrections to the results presented in table 2 are smaller. The APE determinations of refs. [5] 13], obtained with the non-perturbative Clover action at $\beta=6.2$, have not been extrapolated to the continuum limit. In this case, the more extensive analysis by ALPHA-UKQCD [11] shows that the value of the strange quark mass is underestimated by approximately $3 \%$. Finally, in the calculations by APE 13] and JLQCD [14 the conversion from the non-perturbative RI-MOM renormalization scheme to the $\overline{\mathrm{MS}}$ scheme has been done at the $\mathrm{N}^{2} \mathrm{LO}$ in perturbation theory [30, since at the time when these studies have been performed the $\mathrm{N}^{3} \mathrm{LO}$ perturbative calculation of [31] was not available yet. In fig.4, in order to account for the difference between $\mathrm{N}^{2} \mathrm{LO}$ and $\mathrm{N}^{3} \mathrm{LO}$, the results of refs. [13] and [14] have been decreased by $3 \%$.

I would like to emphasize that the previous discussion, besides exploiting the different sources and sizes of systematic errors, also shows that a rather good level of statistical and systematic accuracy has been achieved in the determinations of light quark masses within the quenched approximation. Indeed, the values of $\bar{m}_{s}$ shown in fig. (1) are in remarkable agreement within each other, and even more when the differences in the systematics have been taken into account. From the spread of the results, and accounting for the typical uncertainty on the scale in the quenched case $(\sim 10 \%)$, I obtain as a final average of the lattice results, within the quenched approximation,

$\bar{m}_{s}(2 \mathrm{GeV})^{\mathrm{QUEN}}=(110 \pm 15) \mathrm{MeV}$

This value is shown in fig. 4 , together with the several lattice results and the average value of $\bar{m}_{s}$ quoted by the PDG [23]. Note that the uncertainty in eq. (13) is approximately three times smaller than the one quoted by the PDG, although, in the former, the effect of the quenching approximation has not been taken into account yet.

In the light quark sector, the other quantity, besides $m_{s}$, which is accessible to lattice calculations is the average value of the up and down quark masses, $m_{u d} \equiv\left(m_{u}+m_{d}\right) / 2$. Rather then the value of $m_{u d}$ itself, however, I find more con-
Table 3

Quenched lattice results for the ratio of the strange to the average up-down quark masses.

\begin{tabular}{lc}
\hline & $2 m_{s} /\left(m_{u}+m_{d}\right)$ \\
\hline APE 98 [13 & $24.6 \pm 2.2$ \\
JLQCD 99 [14] & $25.1 \pm 1.7$ \\
CP-PACS 99 26] & $25.3 \pm 1.0$ \\
QCDSF 99 [27] & $23.9 \pm 1.1$ \\
APE 99 [5] & $23.4 \pm 2.4$ \\
CP-PACS 00 [28] & $25.2 \pm 1.0$ \\
\hline
\end{tabular}

The errors are my estimates based on the original data.

venient to consider the ratio of the strange to the average up-down quark masses, because in this ratio many of the systematic uncertainties, coming for instance from the renormalization constants or the choice of the scale, are expected to partially cancel. The values of $m_{s} / m_{u d}$ from the most recent lattice calculations are collected in table 3 and shown in fig. 5. The quoted errors

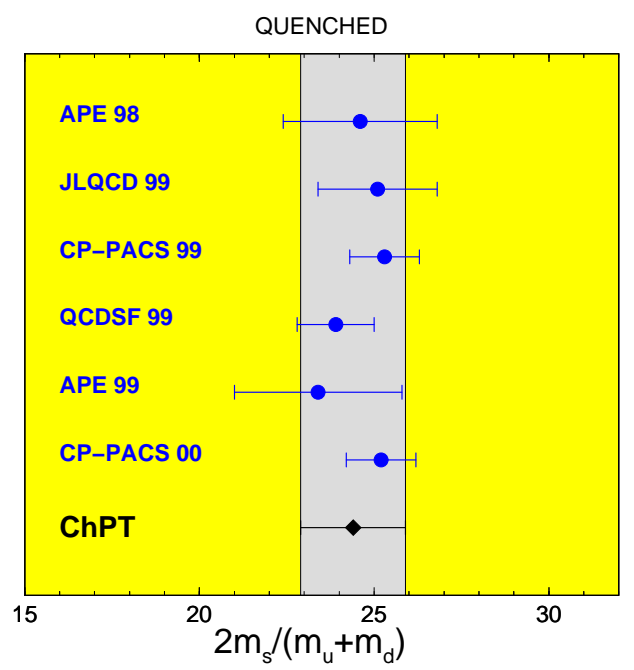

Figure 5. Ratio of the strange to the average updown quark masses as obtained from recent lattice calculations in the quenched approximation (circles). The errors are my estimates based on the original data. The lowest point (diamond) represents the prediction of chiral perturbation theory.

are my estimates based on the original data, be- 
cause the value of this ratio has not been quoted directly in the original papers.

The relevant result, shown in fig. 5, is that the lattice predictions for $m_{s} / m_{u d}$ are in very good agreement with the value $24.4 \pm 1.5$ predicted by chiral perturbation theory [33]. By using this information and eq. (13), I obtain the quenched average:

$\bar{m}_{u d}(2 \mathrm{GeV})^{\mathrm{QUEN}}=(4.5 \pm 0.6) \mathrm{MeV}$.

Finally, I would like to mention that the first (quenched) lattice calculation of $\bar{m}_{u d}$ with overlap fermions has been presented at this conference [34]. The preliminary result is $\bar{m}_{u d}(2 \mathrm{GeV})$ $=5.5(5) \mathrm{MeV}$, in good agreement with the average (14).

\subsection{Unquenched Results}

Several unquenched calculations of light quark masses, with two flavours of dynamical quarks, have been presented at this conference. A compilation of the results, together with details of the simulations, is given in table 4 . With the exception of the SESAM [35] and CP-PACS [28] determinations, all other results presented in the table are still preliminary.

A striking result shown in table is the value of the ratio of the strange to the average updown quark masses obtained by SESAM [35]: $m_{s} / m_{u d} \simeq 55$. This value is larger, by roughly a factor 2, than all other quenched and unquenched lattice determinations (including the quenched result by SESAM [22]), and in disagreement with the prediction of chiral perturbation theory. The reason for such a large value of $m_{s} / m_{u d}$ obtained by SESAM is the way in which the unquenched analysis has been performed. Indeed, as discussed below, by following a different procedure they obtain $m_{s} / m_{u d} \simeq 30$ [22], which is much closer to the expected value. I believe this point is worth to be discussed in details, because it is also of relevance for other unquenched studies.

In numerical simulations with dynamical fermions all physical quantities, as well as the lattice spacing and the critical value of the hopping parameter, are functions of the two parameters entering in the action, namely the coupling constant and the sea quark mass. Theoretical considera- tions suggest that the dependence of the lattice spacing on the value of the sea quark mass should be exponential 40]. Indeed, the lattice scale depends exponentially on the effective coupling constant which, in turn, is affected by the values of quark masses because quarks run in the loops. A large dependence of the lattice spacing on the sea quark mass is in fact observed in actual calculations. For instance, JLQCD [38] finds that, in the range of $K_{\text {sea }}$ used in the simulation (corresponding to $m_{P S} / m_{V} \simeq 0.6-0.8$ ), the inverse lattice spacing varies approximately by $30 \%$, between $\sim 1.5$ and $\sim 2.0 \mathrm{GeV}$. The important point is that such a dependence, if not properly taken into account, may lead to uncontrolled systematic effects in the various extrapolations, in both the sea and valence quark masses.

In order to control these effects, an appropriate procedure for the unquenched analysis has been advocated 40,41]. It consists in performing all the analysis, including the calibration of the lattice spacing and the calculation of the relevant physical quantities, at each fixed value of $K_{\text {sea }}$. The simulations performed at fixed $K_{\text {sea }}$ can be thought of as pseudo-quenched simulations, which come closer to the description of the real world as the sea quark mass approaches its physical value. Only when all the quantities, computed at fixed $K_{\text {sea }}$, are expressed in physical units, the results can be extrapolated in the sea quark mass.

This procedure has been followed by APE 37 in the calculation of the strange quark mass. At two fixed masses of the sea quark, both the strange and the average up-down quark masses have been computed. The results are shown in fig. 6, in terms of the ratios $m_{u d} / m_{K^{*}}$ and $m_{s} / m_{K^{*}}$. Presenting these ratios is equivalent to show the quark masses in physical units. The values of $m_{u d}$ are then extrapolated in the sea quark mass, also computed in physical units, up to the point corresponding to $m_{\text {sea }}=m_{u d}$. Once the physical value of $m_{u d}$ has been determined in this way, a similar extrapolation is performed for the strange mass. From fig. 6 one can see that the final extrapolations in the sea quark mass are indeed smooth.?

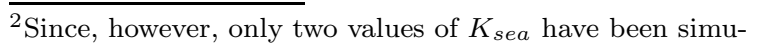


Table 4

Simulation details and physical results of unquenched lattice calculations of light quark masses.

\begin{tabular}{|c|c|c|c|c|c|c|c|c|}
\hline & Action & $a^{-1}$ & $\#\left(\beta, K_{\text {sea }}\right)$ & $Z_{m}$ & \multicolumn{2}{|c|}{$\bar{m}_{s}(2 \mathrm{GeV})$} & $\overline{m_{s} / m_{u d}}$ & $\bar{m}_{s}^{\mathrm{QUEN}} / \bar{m}_{s}^{\mathrm{UNQ}}$ \\
\hline SESAM 98 [35] & Wilson & $2.3 \mathrm{GeV}$ & 4 & $\mathrm{PT}$ & $151(30)$ & $\overline{\left(m_{K, \phi}\right)}$ & $55(12)$ & $1.10(24)$ \\
\hline MILC 99 36 & Fatlink & $1.9 \mathrm{GeV}$ & 1 & $\mathrm{PT}$ & $\begin{array}{l}113(11) \\
125(9)\end{array}$ & $\begin{array}{c}\left(m_{K}\right) \\
\left(m_{\phi}\right)\end{array}$ & $22(4)$ & $1.08(13)$ \\
\hline APE 00 37 & Wilson & $2.6 \mathrm{GeV}$ & 2 & NP-RI & $\begin{array}{l}112(15) \\
108(26)\end{array}$ & $\begin{array}{l}\left(m_{K}\right) \\
\left(m_{\phi}\right)\end{array}$ & $26(2)$ & $1.09(20)$ \\
\hline CP-PACS 00 28] & MF-Clover & $a \rightarrow 0$ & 12 & $\mathrm{PT}$ & $\begin{array}{l}88_{-6}^{+4} \\
90_{-11}^{+5}\end{array}$ & $\begin{array}{c}\left(m_{K}\right) \\
\left(m_{\phi}\right)\end{array}$ & $26(2)$ & $1.25(7)$ \\
\hline JLQCD 0038 & NP-Clover & $2.0 \mathrm{GeV}$ & 5 & $\mathrm{PT}$ & $\begin{array}{l}94(2)^{\dagger} \\
88(3)^{\ddagger} \\
109(4)^{\dagger} \\
102(6)^{\ddagger}\end{array}$ & $\begin{array}{l}\left(m_{K}\right) \\
\left(m_{\phi}\right)\end{array}$ & - & - \\
\hline $\begin{array}{l}\text { QCDSF + } \\
\text { UKQCD } 00 \text { 39 }\end{array}$ & NP-Clover & $2.0 \mathrm{GeV}$ & 6 & $\mathrm{PT}$ & $90(5)$ & $\left(m_{K}\right)$ & $26(2)$ & - \\
\hline
\end{tabular}

${ }^{\dagger}$ From vector WI; ${ }^{\ddagger}$ from axial WI. The errors on the ratios $m_{s} / m_{u d}$ and $\bar{m}_{s}^{\mathrm{QUEN}} / \bar{m}_{s}^{\mathrm{UNQ}}$ are my estimates based on the original data.

In the analysis by SESAM and CP-PACS the pseudoscalar and vector meson masses have been fitted simultaneously in both the sea and the valence quark masses, with all quantities expressed in lattice units. The large dependence of the lattice spacing on the sea quark mass may not be taken properly into account in this way. Indeed, SESAM finds that the value of $m_{s} / m_{u d}$ changes by a factor 2 when the analysis, instead, is performed at fixed values of $K_{\text {sea }}$, as advocated before. Moreover, the result obtained with the analysis performed at fixed $K_{\text {sea }}$ is much closer to the expected value of this ratio. The systematic effects, introduced by the neglecting of the lattice spacing dependence on the sea quark mass, are difficult to evaluate a priori, and depend on the specific values of the parameters used in the calculation. JLQCD, for instance, attempted the chiral extrapolations by using either dimensionless or dimensionful quantities [38] (although considering different orders in the expansions), and

lated by APE, the results should be taken as preliminary. obtained for $m_{s}$ compatible results.

Since unquenched studies of light quark masses have not yet reached the same degree of accuracy achieved in quenched calculations, in order to obtain an estimate of the quenching effect it is convenient to consider directly the ratio between quenched and unquenched results. Some of the systematic errors are expected to cancel in this ratio, when both determinations are performed by using the same action, the same renormalization procedure and the same choice of physical inputs. The results for the ratio $\bar{m}_{s}^{\mathrm{QUEN}} / \bar{m}_{s}^{\mathrm{UNQ}}$ are presented in table 1 . In the very extensive calculation performed by CP-PACS the value $m_{s}^{\mathrm{QUEN}} / m_{s}^{\mathrm{UNQ}}=1.25(8)$ is obtained, suggesting a sizable decrease of the quark mass in the unquenched case. This result, however, is not confirmed by the other (less extensive) studies by SESAM, APE and MILC, which find a decrease, in the unquenched case, rather of the order of $10 \%$. Given these results, and bearing in mind the uncertainties in the analysis methods, I believe that, in order to quote a final estimate of light 


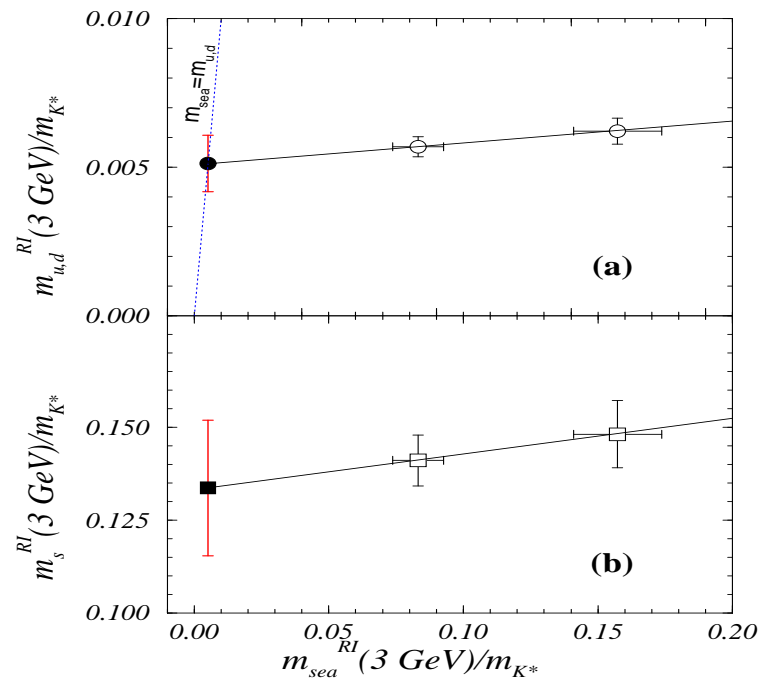

Figure 6. Extrapolation of the average up-down (a) and the strange (b) quark masses, computed at fixed $K_{\text {sea }}$, to the physical value of the sea quark mass, $m_{\text {sea }}=m_{u d}$.

quark masses, it is more appropriate to include the quenching error as an additional systematic uncertainty in the quenched averages (eqs. (13) and (14)), rather than varying the central values. Assuming this error to be of the order of $20 \mathrm{MeV}$ in the case of the strange mass, I then obtain:

$$
\begin{aligned}
& \bar{m}_{s}(2 \mathrm{GeV})=(110 \pm 15 \pm 20) \mathrm{MeV} \\
& \bar{m}_{u d}(2 \mathrm{GeV})=(4.5 \pm 0.6 \pm 0.8) \mathrm{MeV}
\end{aligned}
$$

Note also that in the unquenched case (see table (4) the differences between the determinations of the strange quark mass from $m_{K}$ and $m_{\phi}$, if any, are rather small.

\section{THE b-QUARK MASS}

The $b$-quark cannot be directly simulated on the lattice since its mass is larger than typical values of the ultraviolet cutoff in present lattice calculations $\left(a^{-1} \sim 3 \mathrm{GeV}\right)$. The $b$ mass, however, is also larger than the typical energy scale of strong interactions. Thus, the heavy degrees of freedom of the $b$-quark can be integrated out. Beauty hadrons may be therefore simulated on the lattice in the framework of a low-energy effective theory. Indeed, in the past years, many lattice simula- tions of Heavy Quark Effective Theory (HQET) and Non-Relativistic QCD (NRQCD) have been performed.

Within the effective theory, the mass of a $B$ meson, $M_{B}$, is related to the pole mass of the $b$ quark. Up to higher-order $1 / m_{b}$ corrections, this relation has the form:

$M_{B}=m_{b}^{\text {pole }}+\varepsilon-\delta m$

where $\varepsilon$ is the so-called binding energy, which can be computed non-perturbatively by a numerical simulation, and $\delta m$ is the residual mass, generated by radiative corrections, which can be evaluated in perturbation theory. The perturbative calculation of the residual mass and the nonperturbative (lattice) calculation of the binding energy thus allow a determination of the $b$ pole mass. The result can be then translated into the $\overline{\mathrm{MS}}$ mass, $\bar{m}_{b}$, by using perturbation theory.

An important observation, concerning this procedure, is that the binding energy $\varepsilon$ is not a physical quantity, and it is affected by power divergences proportional to the inverse lattice spacing, $1 / a$. These divergences are canceled by similar singularities in the residual mass $\delta m$. Moreover, the pole mass $m_{b}^{\text {pole }}$, and consequently $\delta m$, are also affected by renormalon singularities, which introduce in their definitions an uncertainty of the order of $\Lambda_{Q C D}$ 42, 43. These singularities are then canceled by the perturbative series relating the pole mass and the $\overline{\mathrm{MS}}$ mass 44 . As a result, the $\overline{\mathrm{MS}}$ mass, $\bar{m}_{b}$, is a finite, well defined, shortdistance quantity. In the actual calculation, however, since the residual mass is computed up to a finite order in perturbation theory, only a partial cancellation of both power divergences and the renormalon singularities occurs. For this reason, it is crucial to compute the residual mass $\delta m$ up to the highest possible order in perturbation theory. Moreover, because of the incomplete cancellation of power divergences, the lattice spacing in such calculations cannot be taken too small.

At present, the most accurate determination of $\delta m$ has been obtained in the framework of HQET. In terms of the lattice bare coupling constant, the perturbative expansion can be written as:

$a \delta m=C_{1} \alpha_{0}+C_{2} \alpha_{0}^{2}+C_{3} \alpha_{0}^{3}+\ldots$ 
The two-loop coefficient, $C_{2}$ has been derived analytically, by Martinelli and Sachrajda 45], from the study of the asymptotic behaviour of Wilson loops. In the quenched case $\left(N_{f}=0\right)$, also the coefficient $C_{3}$ has been evaluated, by Di Renzo and Scorzato [46], using the method of numerical stochastic perturbation theory. An important check of this approach is obtained from the comparison of their numerical results for the first two coefficients, $C_{1}=2.09(4)$ and $C_{2}=10.7(7)$, with the analytically known values: $C_{1}=2.1173$ and $C_{2}=11.152$ 45. The value obtained for the third coefficient, $C_{3}=86.2(5)$, is also consistent with the determination by Lepage et al. 447, $C_{3}=81(2)$, obtained, within a completely different approach, by fitting the results of small coupling Monte Carlo calculations.

These combined theoretical efforts allow a determination of the $b$-quark mass which is accurate, in the quenched approximation, up to the $\mathrm{N}^{3} \mathrm{LO}$. Two independent results have been obtained so far:

$\bar{m}_{b}{ }^{\text {QUEN }}=(4.30 \pm 0.05 \pm 0.05) \mathrm{GeV}$

$\bar{m}_{b}^{\text {QUEN }}=(4.34 \pm 0.03 \pm 0.06) \mathrm{GeV}$ 49

nicely in agreement within each other. Remarkably, the two determinations have been derived using different approaches. The APE result (19) has been obtained within HQET, while the determination (20), by Collins et al., is obtained from the NRQCD study of the $B$-meson spectrum in the static limit. The last error in eqs. (19) and (20) represents the residual uncertainty due to the neglecting of higher orders in the perturbative expansion of $\delta m$. For comparison, this uncertainty was estimated to be of the order of $200 \mathrm{MeV}$ at NLO [50] and $100 \mathrm{MeV}$ at $\mathrm{N}^{2} \mathrm{LO}$ [45] respectively.

Results compatible with those in eqs. (19) and (20) have been obtained from the study of the $B$ and the $\Upsilon$ spectrum with NRQCD [51,52]. In this case, however, only the coefficient $C_{1}$ is known, so that the achieved accuracy is at NLO. These results are thus affected by a larger perturbative uncertainty, which can be estimated, on the basis of a renormalon analysis [44, to be of the order of $100 \mathrm{MeV}$.

The first unquenched calculation of the $b$-quark mass has been performed by APE this year [40].
The perturbative accuracy is at $\mathrm{N}^{2} \mathrm{LO}$, since the three-loop coefficient, in the expansion of $\delta m$, is yet unknown in the unquenched case. Their result,

$\bar{m}_{b}=(4.26 \pm 0.06 \pm 0.07) \mathrm{GeV}$.

represents to date the most accurate determination of the $b$-quark mass from lattice QCD calculations. Remarkably, the relative uncertainty is reduced at the level of $2 \%(90 \mathrm{MeV})$.

A preliminary analysis 49] performed in the static limit with NRQCD indicates a decrease of $\bar{m}_{b}$, in the unquenched case, by approximately 70 $\mathrm{MeV}$. Combined with the quenched determination (20), this result leads to an estimate of the $b$-quark mass in good agreement with (21).

\section{CONCLUSIONS}

Significant progresses in lattice calculations of light and heavy quark masses have been allowed by the introduction of improved actions and nonperturbative renormalization techniques.

Current determinations of light quark masses have reached a good level of statistical and systematic accuracy, which is of the order of $10 \%$ within the quenched approximation. The main source of uncertainty, in this case, is due to the quenched approximation. Several unquenched results, with two flavours of dynamical quarks, have been presented at this conference. The effect of quenching is found to be in the range of $10-20 \%$, but further investigations are required to obtain a firmer estimate.

I have not reported, in this review, lattice calculations of the charm quark mass, since recent results for this quantity are missing. The charm mass is of great phenomenological interest since it enters, through the heavy quark expansion, the theoretical predictions of several cross sections and decay rates. For this reasons, new lattice calculations with the non-perturbatively improved action and non-perturbative renormalization techniques is highly recommended.

Enormous progresses have been achieved in lattice calculations of the bottom quark mass. The present accuracy is at $\mathrm{N}^{3} \mathrm{LO}$ in the quenched approximation, and at $\mathrm{N}^{2} \mathrm{LO}$ in the unquenched 
case. The relative uncertainty is reduced at the impressive level of $2 \%$.

Final averages of lattice calculations for quark masses have been given in eqs. (15), (16) and (21). By combining statistical and systematic errors, these correspond to:

$$
\begin{aligned}
& \bar{m}_{u d}(2 \mathrm{GeV})=(4.5 \pm 1.0) \mathrm{MeV} \\
& \bar{m}_{s}(2 \mathrm{GeV})=(110 \pm 25) \mathrm{MeV} \\
& \bar{m}_{b}\left(\bar{m}_{b}\right)=(4.26 \pm 0.09) \mathrm{GeV}
\end{aligned}
$$

\section{ACKNOWLEDGEMENTS}

I thank D. Becirevic, C. Bernard, R. Burkhalter, C. Davies, F. Di Renzo, V. Gimenez, L. Giusti, T. Kaneko, H. Leutwyler, K.F. Liu, G. Martinelli, R. Sommer, M. Wingate and H. Wittig for discussions and private communications.

\section{REFERENCES}

1. K. Jansen et al., Phys. Lett. B372 (1996) 275, hep-lat/9512009;

M. Lüscher et al., Nucl. Phys. B478 (1996) 365, hep-lat/9605038;

M. Lüscher et al., Nucl. Phys. B491 (1997) 323, hep-lat/9609035;

M. Lüscher et al., Nucl. Phys. B491 (1997) 344, hep-lat/9611015.

2. G. Martinelli et al., Nucl. Phys. B445 (1995) 81, hep-lat/9411010.

3. ALPHA Collaboration, S. Capitani et al., Nucl. Phys. B544 (1999) 669, hep-lat/ 9810063.

4. M. Bochicchio et al., Nucl. Phys. B262 (1985) 331.

5. APE Collaboration, D. Becirevic et al., Phys. Rev. D61 (2000) 114507, hep-lat/9909082.

6. K. Lane, Phys. Rev. D10 (1974) 2605;

H.D. Politzer, Nucl. Phys. B117 (1976) 397;

P. Pascual and E. de Rafael, Zeit. Phys. C12 (1982) 127.

7. G. Martinelli et al., EDINBURGH 96/28, unpublished; C. Dawson et al., Nucl. Phys. Proc. Suppl. 63 (1998) 877, hep-lat/ 9710027.

8. M. Guagnelli et al., DESY 00-131, hep-lat/ 0009021.
9. T. Bhattacharya et al., LAUR-00-3538, hep-lat/0009038.

10. T. Bhattacharya and R. Gupta, Nucl. Phys. Proc. Suppl. 63 (1998) 95, hep-lat/9710095.

11. ALPHA and UKQCD Collaboration, J. Garden et al., Nucl. Phys. B571 (2000) 237, hep-lat/9906013.

12. S. Sint, this proceedings.

13. APE Collaboration, D. Becirevic et al., Phys. Lett. B444 (1998) 401, hep-lat/9807046.

14. JLQCD Collaboration, S. Aoki et al., Phys. Rev. Lett. 82 (1999) 4392, hep-lat/9901019.

15. RBC Collaboration, M. Wingate, Nucl. Phys. Proc. Suppl. 83 (2000) 221, hep-lat/ 9909101.

16. J. Cudell, A. Le Yaouanc and C. Pittori, Phys. Lett. B454 (1999) 105, hep-lat/ 9810058.

17. D. Becirevic et al., Nucl. Phys. Proc. Suppl. 83 (2000) 863, hep-lat/9909039.

18. L. Giusti and A. Vladikas, Phys. Lett. B488 (2000) 303, hep-lat/0005026.

19. K.G. Chetyrkin, Phys.Lett. B404 (1997) 161, hep-ph/9703278.

20. J.A.M. Vermaseren, S.A. Larin and T. van Ritbergen, Phys. Lett. B405 (1997) 327, hep-ph/9703284.

21. C.R. Allton et al., Nucl. Phys. B431 (1994) 667, hep-ph/9406263.

22. SESAM Collaboration, N. Eicker et al., Phys. Lett. B407 (1997) 290, hep-lat/9704019.

23. Review of Particle Physics, Eur. Phys. J. C15, 1 (2000).

24. L. Lellouch, E. de Rafael and J. Taron, Phys. Lett. B414 (1997) 195, hep-ph/9707523.

25. D. Becirevic, private communication.

26. CP-PACS Collaboration, S. Aoki et al., Phys. Rev. Lett. 84 (2000) 238, hep-lat/9904012.

27. QCDSF Collaboration, M. Gockeler et al., Phys. Rev. D62 (2000) 054504, hep-lat/ 9908005.

28. CP-PACS Collaboration, A. Ali Khan et al., UTCCP-P-85, hep-lat/0004010; see also R. Burkhalter, this proceedings, hep-lat/ 0010078.

29. RBC Collaboration, M. Wingate, this proceedings, hep-lat/0009023.

30. E. Franco and V. Lubicz, Nucl. Phys. B531 
(1998) 64, hep-ph/9803491.

31. K.G. Chetyrkin and A. Retey, Nucl. Phys. B583 (2000) 3, hep-ph/9910332.

32. R. Sommer, Nucl. Phys. B411 (1994) 839, hep-lat/9310022.

33. H. Leutwyler, Phys. Lett. B378 (1996) 313, hep-ph/9602366; see also H. Leutwyler, this proceedings, hep-ph/0011049.

34. S.J. Dong et al., hep-lat/0006004; see also K.F. Liu, this proceedings, hep-lat/ 0011072

35. SESAM Collaboration, N. Eicker et al., Phys. Rev. D59 (1999) 014509, hep-lat/9806027. See also 22.

36. MILC Collaboration, C. Bernard, private communication.

37. APE Collaboration, D. Becirevic, V. Gimenez, L. Giusti, V. Lubicz and G. Martinelli, unpublished.

38. JLQCD Collaboration, T. Kaneko, this proceedings, hep-lat/0010086.

39. QCDSF and UKQCD Collaboration, D. Pleiter, this proceedings, hep-lat/0010063.

40. APE Collaboration, V. Gimenez et al., JHEP 0003 (2000) 018, hep-lat/0002007.

41. UKQCD Collaboration, C.R. Allton et al., Phys. Rev. D60 (1999) 034507, hep-lat/ 9808016.

42. M. Beneke and V.M. Braun, Nucl. Phys. B426 (1994) 301, hep-ph/9402364.

43. I.I. Bigi et al., Phys. Rev. D50 (1994) 2234, hep-ph/9402360.

44. G. Martinelli and C.T. Sachrajda, Nucl. Phys. B478 (1996) 660, hep-ph/9605336.

45. G. Martinelli and C.T. Sachrajda, Nucl. Phys. B559 (1999) 429, hep-lat/9812001.

46. F. Di Renzo and L. Scorzato, this proceedings, hep-lat/0010064.

47. G.P. Lepage et al., Nucl. Phys. Proc. Suppl. 83 (2000) 866, hep-lat/9910018.

48. V. Gimenez, private communication.

49. C. Davies, private communication. See also S. Collins, hep-lat/0009040.

50. V. Gimenez, G. Martinelli and C.T. Sachrajda, Phys. Lett. B393 (1997) 124, hep-lat/ 9607018.

51. A. Ali Khan et al., Phys. Rev. D62 (2000) 054505, hep-lat/9912034.
52. NRQCD Collaboration, C.T.H. Davies, et al., Nucl. Phys. Proc.Suppl. 73 (1999) 339, hep-lat/9809177. 